\title{
PARTITION FUNCTION FOR ONE ELECTRON IN A PLASMA; SAHA EQUATION
}

\author{
R. DEKEYSER * \\ Instituut voor Theoretische Fysica, Rijksuniversiteit, Utrecht
}

Received 21 April 1965

Experimentalists working with flames [1] and astronomers [2] often encounter situations where they would like to make use of the partition function for a single electron in a plasma, including the bound states.

To be more specific, let us consider a hydrogen plasma, consisting of $N$ electrons and $N$ protons, contained in a volume $V$. It is a well known fact that in the partition function the sum over all bound states of the hydrogen atom

$$
\sum_{n=1}^{\infty} n^{2} \exp \left[-\frac{\beta E_{\mathrm{o}}}{n^{2}}\right]
$$

diverges strongly. Here $\beta=1 / k T$ and $E_{0}$ is the lowest hydrogen energy level

$$
E_{\mathrm{O}}=-m e^{4} / 2 \hbar^{2} .
$$

This divergence has been understood a long time ago: it is due to the very high orbits, that are unphysical when they contain several other ions, and certainly when they exceed the walls of the volume. Several attempts [3] have been made to give a quantitatively correct description, but none of them is really satisfactory.

We calculated the partition function of an electron in a hydrogen plasma, by dividing it in three parts. The possible situations for the electron under consideration in each part are:

A) The bound states that the electron can form with any of the $N_{\mathrm{i}}$ ionized protons, but only up to a certain maximum energy

$$
-A=-E_{\mathrm{o}} / \nu^{2}
$$

corresponding to a principal quantum number $\nu$.

B) The situations where the electron comes closer to any of the $N_{\mathrm{i}}$ ionized protons than a distance $e^{2} / A$, but with an energy higher than the maximum $-A$; this imposes a minimum condition on the electron momentum.
C) The situations where our electron is farther away than $e^{2 / A}$ from each of the $N_{\mathrm{i}}$ protons. For the moment, we suppose that we know the degree of ionization $N_{\mathrm{i}} / N$. Parts B) and C) are calculated classically with the formula [4]

$Z=h^{-3} \int \mathrm{d} E \int \mathrm{d} \boldsymbol{p} \int \mathrm{d} \boldsymbol{x} \delta\left[E-p^{2} / 2 m-U(x)\right] \mathrm{e}^{-\beta E}$,

where $h$ is Planck's constant, $E$ the energy of the electron, $p$ its momentum and $U$ its potential energy. The domains of integration are to be adapted to each case. In B) we take only the potential energy from the proton to which the electron is so close. In C) we must take the sum of the potential energies of our electron coming from all other electrons and protons, integrated over their possible configurations with an appropriate weight function.

Now it appears to be possible for any usual plasma to make such a division, satisfying at the same time the conditions that the quantummechanical energy levels corresponding to C) lie close enough together to allow us to treat them classically, and that the largest distance between electron and proton in A) and B) are small enough in comparison with the mean interparticle distance to allow to forget about the rest of the plasma. At the same time we can satisfy the condition

$$
A \beta \ll 1,
$$

which yields us a parameter for developments in series. The calculations of the partition function in this way are rather complicated, and we refer to an other work [5], where we calculated the partition function for the whole plasma, following the same basic ideas. We only state the result:

\footnotetext{
* Aangesteld navorser van het Belgisch Nationaal Fonds voor Wetenschappelijk Onderzoek.
} 


$$
\begin{aligned}
Z= & V\left(\begin{array}{c}
m \\
2 \pi \beta \hbar^{2}
\end{array}\right)^{\frac{3}{2}}\left(1+\frac{2}{3} \beta e^{2} \kappa\right)+ \\
& +N_{\mathrm{i}}\left[\sum_{n=1}^{\nu} n^{2} \exp \left(\frac{\beta m e^{4}}{2 n^{2} \hbar^{2}}\right)-\frac{1}{3} \nu^{3}-\frac{\beta \nu m e^{4}}{2 \hbar^{2}}-\cdots\right]
\end{aligned}
$$

Under the assumed conditions, $v$ must be a large number; it is then easy to see that the part between the square brackets is insensitive to the choice of $\nu$, up to the first order in $A \beta$. Let us call this square bracket term $Z_{\mathrm{B}} \exp -\beta E_{\mathrm{O}}$, the bound part of the partition function. The probability for an electron to be ionized or free is proportional to the corresponding part of the partition function. This means that

$$
\frac{N_{\mathrm{i}}}{N-N_{\mathrm{i}}}=\frac{V(m / 2 \pi \beta \hbar 2)^{\frac{3}{2}}\left(1+\frac{2}{3} \beta e^{2} \kappa\right)}{N_{\mathrm{i}} Z_{\mathrm{B}} \exp \beta\left|E_{\mathrm{O}}\right|},
$$

which is the well known Saha equation. The factor $\left(1+\frac{2}{3} \beta e^{2} \kappa\right)$ can be interpreted as a correction to the ionization potential. An exact examination of the partition function for the whole plasma, however, reveals that the lowering in the ionization potential is $k e^{2}$, without the factor $\frac{2}{3}$ (see ref. 5).

I wish to thank Professor N. G. Van Kampen for the suggestion of the problem and for helpful discussions.

\section{References}

1. T.Hollander, Self-absorption, ionization and dissociation of metal vapour in flames; Thesis, Utrecht 1964.

2. H. Hubenet, Recherches Astronomiques de l'Observatoire d'Utrecht 16 (1960).

3. A survey of these earlier attempts was given by G. Ecker and W. Weizel, Ann. Phys.17 (1956) 126.

4. R.H. Fowler and E.A.Guggenheim, Statistical Thermodynamics, Cambridge University Press 1939 , p. 74 .

5. R. Dekeyser, Physica, to be published. 